\title{
Shutdown Dose Rate Analysis with CAD Geometry, Cartesian/Tetrahedral Mesh, and Advanced Variance Reduction
}

\author{
Elliott D. Biondo ${ }^{\mathrm{a}, *}$, Andrew Davis ${ }^{\mathrm{a}}$, Paul P.H. Wilson ${ }^{\mathrm{a}}$ \\ ${ }^{a}$ University of Wisconsin - Madison, 1500 Engineering Drive, Madison, WI 53706
}

\begin{abstract}
In fusion energy systems (FES) high-energy neutrons born from burning plasma activate system components to form radionuclides. The biological dose rate that results from photons emitted by these radionuclides after shutdown - the shutdown dose rate (SDR) - must be quantified for maintenance planning. This can be done using the Rigorous Two-Step (R2S) method, which involves separate neutron and photon transport calculations, coupled by a nuclear inventory analysis code. The geometric complexity and highly-attenuating configuration of FES motivates the use of CAD geometry and advanced variance reduction for this analysis.

An R2S workflow has been created with the new capability of performing SDR analysis directly from CAD geometry with Cartesian or tetrahedral meshes and with biased photon source sampling, enabling the use of the Consistent Adjoint Driven Importance Sampling (CADIS) variance reduction technique. This workflow has been validated with the Frascati Neutron Generator (FNG)-ITER SDR benchmark using both Cartesian and tetrahedral meshes and both unbiased and biased photon source sampling. All results are within $20.4 \%$ of experimental values, which constitutes satisfactory agreement. Photon transport using CADIS is demonstrated to yield speedups as high as $8.5 \cdot 10^{5}$ for problems
\end{abstract}

\footnotetext{
* Corresponding author

Email addresses: biondo@wisc.edu (Elliott D. Biondo), davisa@engr.wisc.edu (Andrew Davis), wilsonp@engr.wisc.edu (Paul P.H. Wilson)
}

Preprint submitted to Fusion Engineering and Design

February 24, 2016

(C) 2016. This manuscript version is made available under the Elsevier user license http://www.elsevier.com/open-access/userlicense/1.0/ 
using the FNG geometry.

Keywords: shutdown dose rate, $\mathrm{CAD}$, tetrahedral mesh, variance reduction

\section{Introduction}

In experimental and conceptual fusion energy systems (FES) such as ITER [1] high-energy (14.1 MeV) neutrons are born from D-T fusion reactions in burning plasma. These neutrons penetrate deep into system components, producing 5 radionuclides. These radionuclides persist after neutron-producing operations cease and may emit high-energy $(0.01-10 \mathrm{MeV})$ photons as they decay. The potential biological dose rate resulting from these photons after shutdown - the shutdown dose rate (SDR) - must be quantified as a function of position and time after shutdown in order to ensure occupational safety during maintenance operations.

This quantification can be done computationally using the Rigorous TwoStep (R2S) method 2. With the R2S method, neutron transport and photon transport simulations are done separately, coupled by a nuclear inventory analysis code. For FES analysis, Monte Carlo (MC) radiation transport is typically used due to the large physical size and geometric complexity of FES and the importance of particle streaming. A dedicated nuclear inventory analysis code is used due to the complexity of the network of reaction pathways that produce important radionuclides. The coupling process requires multi-group neutron fluxes and geometry/material descriptions to be defined in discrete regions such as geometry cells or a mesh.

In the mesh-based R2S procedure [3, represented in Figure 1, a neutron transport simulation provides mesh-based multi-group neutron fluxes. The continuous representation of the geometry used for $\mathrm{MC}$ transport is then discretized in order to create a mesh-based description of materials. Using the mesh-based materials and neutron fluxes and a known irradiation scenario, a nuclear inventory analysis code is used to determine the energy-wise photon emission density within each mesh volume element for post-shutdown decay times of interest. 


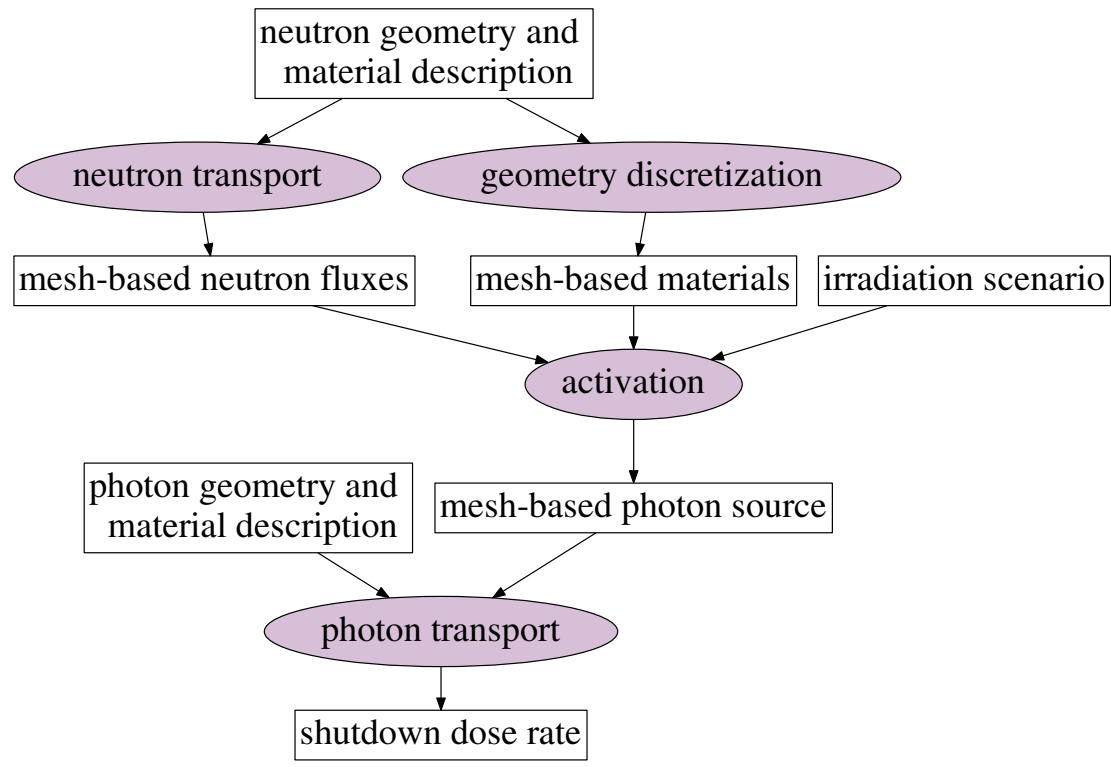

Figure 1: Mesh-based R2S procedure for quantifying the shutdown dose rate. White rectangles represent data and purple ovals represent code execution.

These photon emission density distributions are then used to define sources for separate MC photon transport simulations (one per decay time). Photon fluxes 30 are tallied at positions of interest and flux-to-dose-rate conversion factors are used to estimate the biological dose rate.

FES geometries typically consist of a tremendous number of individual geometric components, many of which may be defined by high-order surfaces. This geometric complexity causes challenges for MC radiation transport simulations which historically use Constructive Solid Geometry (CSG) to represent geometry. In the case of ITER, Computer Aided-Design (CAD) models are created for device assembly. The ability to perform radiation transport on these preexisting models without the laborious process of translating into CSG reduces human effort and design iteration time. This can be accomplished with the

40 Direct Accelerated Geometry Monte Carlo (DAGMC) 4 toolkit (within the Mesh Oriented dAtaBase (MOAB) [5] library), which performs the CAD geom- 
etry queries required for MC particle tracking. DAGMC can be incorporated into industry-standard MC codes allowing radiation transport to be done directly on CAD geometry. DAGMC has been incorporated into MCNP5 to form DAG-MCNP5, which has long been used for high-resolution analysis of FES [6].

Though geometry can be represented continuously for MC transport, a discrete geometry representation is required for the R2S activation step. Discretizing the geometry onto a Cartesian mesh usually results in mesh volume elements that contain multiple geometry cells that may contain different materials. These materials may have significantly different transport cross sections and activation properties, so aggregate treatment may introduce significant systematic error into calculated photon emission densities. It is therefore desirable to tally neutron fluxes and carry out R2S activation with a conformal mesh, with each mesh volume element containing exactly one geometry cell. In cases where conformal meshes are not available, the "cell-under-voxel" can be used to obtain similar results [7. Using this approach the neutron flux is tallied separately for each cell region within a mesh volume element (i.e. for each cell-undervoxel). Activation and photon source sampling are then carried out treating each cell-under-voxel independently.

Tetrahedral mesh can be generated such that mesh volume elements conform to geometry cells, within some tolerance. For the mesh to be deemed conformal, analysts must choose an appropriate tolerance, such that any effects that arise from tetrahedral elements containing multiple geometry cells can be considered negligible. Tetrahedral mesh capabilities are available with MCNP6 8 and DAG-MCNP5. MCNP6 allows for MC radiation transport on tetrahedral mesh generated from CAD geometry (i.e. not directly on CAD geometry). Tetrahedral R2S analysis has been demonstrated with MCNP6 9]. In contrast, DAG-MCNP5 supports transport directly on CAD geometry with superimposed tetrahedral mesh tallies. CUBIT CAD software [10] is used to automatically generate conformal (within some tolerance) tetrahedral mesh from CAD geometry for this purpose. This decoupling of geometry representation and tally mesh is advantageous for R2S analysis, because only portions of the geometry relevant 
to activation must be tallied, which can greatly reduce computer memory requirements. Preliminary analysis has also shown that the direct CAD transport approach may be significantly faster than the tetrahedral mesh transport approach [11, further motivating the application of direct CAD transport to R2S analysis.

Though MC radiation transport is required for detailed FES analysis, the highly-attenuating configurations of FES shielding problems require the use of $\mathrm{MC}$ variance reduction in order to achieve satisfactory MC tally convergence with reasonable computer processor times. In contrast, deterministic radiation transport techniques can provide computationally inexpensive results with additional systematic error (with respect to MC) introduced by spatial, angular, and energy discretization. The Consistent Adjoint Driven Importance Sampling (CADIS) method [12] is a hybrid MC/deterministic transport technique that delivers accurate $\mathrm{MC}$ transport results, accelerated by a deterministic transport pre-processing step. For a given detector response function (tally), a deterministic adjoint transport calculation is carried out using the detector as an adjoint source. The resulting adjoint flux distribution is used to calculate $\mathrm{MC}$ weight windows and source biasing parameters that optimize $\mathrm{MC}$ transport with respect to the detector. Additionally, the Forward-Weighted (FW)-CADIS method can be used to optimize transport in respect to multiple tallies or mesh tallies [13. This is done by generating an adjoint source for use with the standard CADIS method. This adjoint source is defined on the basis of an additional deterministic estimate of the forward flux. FW-CADIS can be used for global variance reduction by optimizing radiation transport in respect to a mesh tally that covers the entire region of interest. The CADIS and FW-CADIS methods have been shown to dramatically speed up MC radiation transport calculations for fusion neutronics applications [14].

In this work, a mesh-based R2S workflow was created with the novel capability of automatically performing SDR analysis directly on CAD geometry using either Cartesian or tetrahedral mesh. This is accomplished using DAGMCNP5 for radiation transport. This work also demonstrates the novel appli- 
cation of advanced variance reduction techniques to the photon transport step of this workflow is provided elsewhere [17. A neutron transport simulation is first done using DAG-MCNP5. Neutron fluxes are tallied in the 175 group VITAMIN-J group structure using a standard MCNP5 mesh tally or a DAGMCNP5 tetrahedral mesh tally covering the geometry of interest for neutron 130 the CAD geometry, and the irradiation scenario. 
For Cartesian meshes, each mesh volume element may contain multiple geometry cells which may consist of different materials. The aggregate material composition for each mesh volume element is ascertained using a ray tracing technique (also facilitated by DAGMC) which has been shown to be more computationally efficient than point-sampling techniques [18. Conformal tetrahedral mesh is generated with CUBIT. Provided that a sufficiently high resolution is used, the approximation that each mesh volume element consists of a single material can be made, and DAGMC is used to simply query the material at the center of the mesh volume element. Once PyNE irradiation_setup is complete, ALARA can be run without any further modification. The photon emission densities output by ALARA for each mesh volume element are then converted into a mesh-based representation using the PyNE photon_sampling_setup function in the PyNE r2s module.

The PyNE photon_sampling_setup function produces a photon emission density mesh for each decay time of interest. These meshes are then used as sources for DAG-MCNP5 photon transport, allowing the photon biological dose rate to be tallied. This is done using the PyNE source_sampling module. This module, written in $\mathrm{C}++$ using $\mathrm{MOAB}$, provides a generic method for sampling the initial position and energy of $\mathrm{MC}$ particles from a Cartesian or tetrahedral mesh source. MCNP5 ships with a customizable source subroutine: source.F90. A source.F90 file was written, incorporating the functionality of PyNE source_sampling, and was then compiled into DAG-MCNP5.

The PyNE source_sampling module first reads the mesh-based energy-wise particle emission densities and creates a probability density function (PDF) with each PDF bin representing the probability that a particle is born within each discrete region of position/energy phase space. This PDF is converted into an alias table [19]. This pre-processing step is performed in $O\left(N^{2}\right)$ computational time complexity, where $N$ is the number of PDF bins, and allows for $O(1)$ sampling of the PDF. MC radiation transport typically involves the simulation of $10^{6}-10^{12}$ particles, so this expensive setup step is well-justified. The alias table is then sampled using random numbers supplied by MCNP5. A phase 
space bin is first selected and the exact birth position and energy are then sampled uniformly within the selected bin. Uniform sampling of tetrahedral mesh volume elements is accomplished using a method described in Rocchini et al [20].

The PyNE source_sampling module also allows for biased random sampling of mesh-based sources, which can be used for MC variance reduction. This is done by supplying mesh-based biased particle emission densities (in addition to unbiased emission densities). These biased emission densities can be produced using methods such as CADIS/FW-CADIS. The PyNE source_sampling routine will sample these biased emission densities and adjust the statistical weight of the particles accordingly. The routine can also automatically generate the 175 biased particle emission densities necessary for uniform sampling (i.e. equiprobable particle birth in any position) for a given unbiased particle emission density mesh.

\section{Benchmarking}

Comparing simulation results to experimental results (i.e. benchmarking) is an essential step for the validation of computational tools. The PyNE R2S workflow has been validated with the FNG ITER shutdown dose rate benchmark. This experimental benchmark was designed to emulate the irradiation of the ITER outer vacuum vessel region [16].

The FNG experimental assembly (shown in Figure 2) consists of a $99 \mathrm{~cm} \times$ $71.83 \mathrm{~cm} \times 98.4 \mathrm{~cm}$ block of stainless steel 316 (SS316) and water-equivalent polymethyl methacrylate (PMMA). The neutron generator beam is directed down a $1.37 \mathrm{~cm}$ radius cylindrical channel perpendicular to the $99 \mathrm{~cm} \times 98.4 \mathrm{~cm}$ face of the block. This channel is $22.57 \mathrm{~cm}$ long and terminates at an inner cavity. This inner cavity also opens to a lateral access port. In one portion of the experiment ("campaign 2"), the experimental assembly was irradiated with a series of pulses of $14 \mathrm{MeV}$ neutrons for approximately 14 hours over the course of approximately 30 hours with an SS316 plug in the access port. The plug 
was then removed and a tissue-equivalent scintillator (dosimeter) was placed in the central cavity and the biological dose rate was measured at 19 decay times ranging from 1.22 hours to 19.8 days. The authors of the benchmark also provided computational results for the SDR for each of these 19 decay times using the R2S and Direct One-Step (D1S) methods [16]. For this computational benchmarking, the pulsed irradiation scenario was approximated with 4 ontimes.

In order to benchmark the PyNE R2S workflow, computational results for the FNG ITER benchmark were obtained using multiple meshes and photon sampling modes. MCNP4c models of the neutron and photon FNG geometries were obtained from the SINBAD [21] benchmark suite and converted into CAD models using the MCNP2CAD tool [22].

Neutron transport was done with DAG-MCNP5 with ENDF/B-VII nuclear data [23]. Neutron transport was done using weight windows generated with the MAGIC method 24] (implemented within the PyNE variance_reduction module) with $1.6 \cdot 10^{8}$ particles simulated. Neutron fluxes were tallied on a $73 \times 58 \times 65$ Cartesian mesh $(275,210$ mesh volume elements $)$ and also a conformal tetrahedral mesh with 244,710 mesh volume elements, generated using CUBIT. The resulting neutron flux distributions are shown in Figure 3. The distribution of relative errors for all position/energy phase space bins is shown in Figure 4 Nearly all phase space bins $(99.66 \%$ and $99.60 \%$ for Cartesian and tetrahedral meshes respectively) had relative errors less than 0.2 . The small fraction of phase space bins with relative errors greater than 0.2 are high-energy bins far from the neutron source position.

Nuclear inventory analysis was done with ALARA using FENDL3.0/A [25] transmutation and photon source libraries. The same approximated irradiation scenario used in the original computational benchmark was applied and a 24group photon group structure was used (the same as used by FISPACT nuclear inventory analysis code [26]). Photon emission density distributions were obtained for each of the 19 decay times. The photon emission density distributions using Cartesian and tetrahedral meshes for the first decay time are shown in 


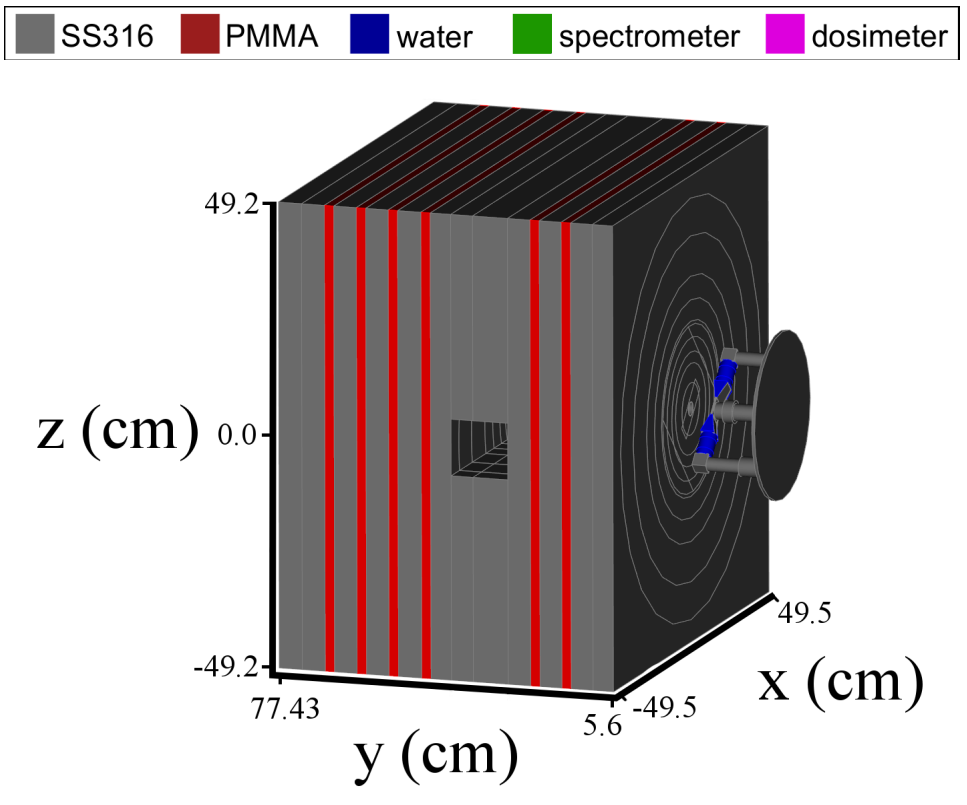

(a) full geometry

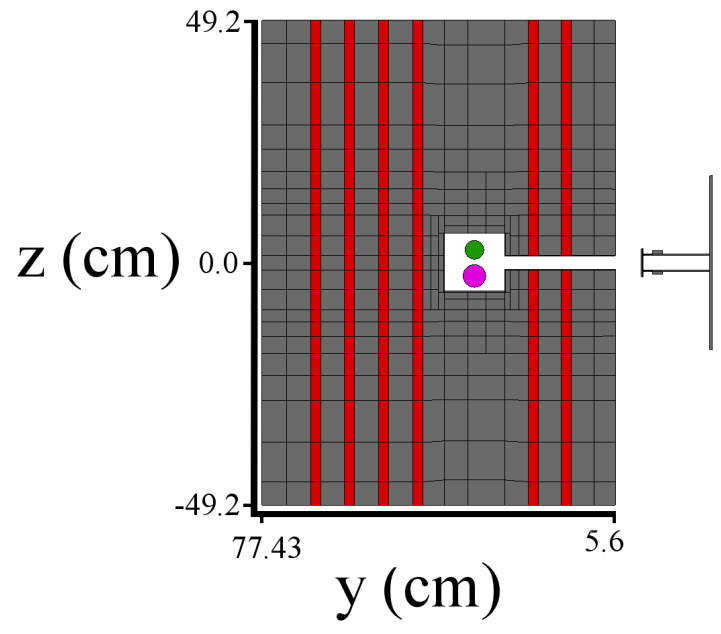

(b) $y$ - $z$ slice through $x=0$

Figure 2: CAD representation of the FNG geometry with the lateral access port open and detectors in the central cavity.

Figure 5 .

All photon transport was done with DAG-MCNP5 with ENDF/B-VII nu- 


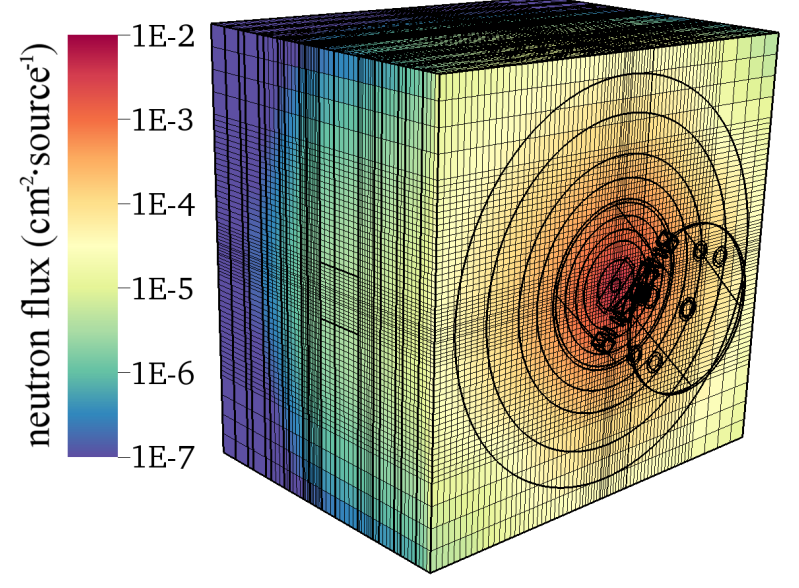

(a) Cartesian mesh

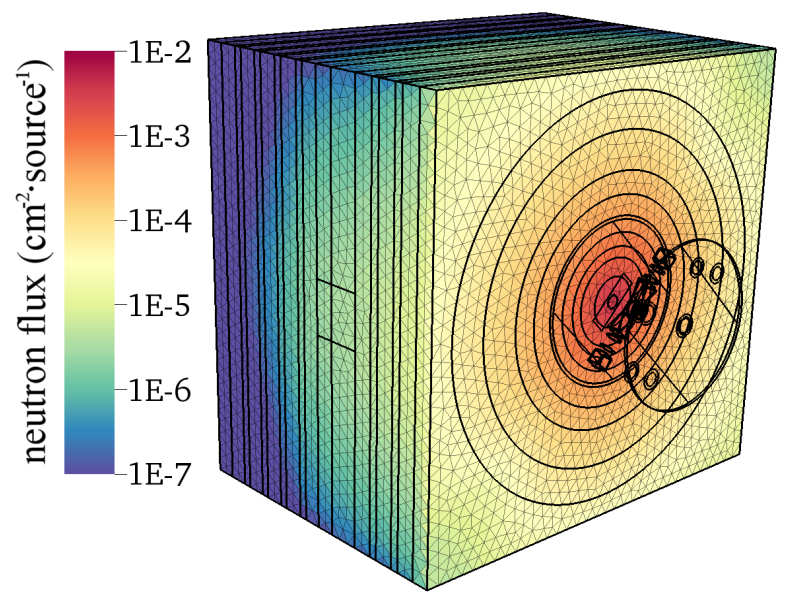

(b) Tetrahedral mesh

Figure 3: Total neutron flux distributions using Cartesian and tetrahedral meshes. Thick black lines represent geometry cells and thin black lines represent mesh volume elements.

clear data. For each of the 19 decay times, photon transport was done in analog (i.e. no variance reduction except implicit capture) and with CADIS biasing and weight windows. Variance reduction is not imperative for the photon transport step in this problem; CADIS was used only for the purpose of validating the 230 biased source sampling feature.

The CADIS method for generating weight windows and biased sources is 


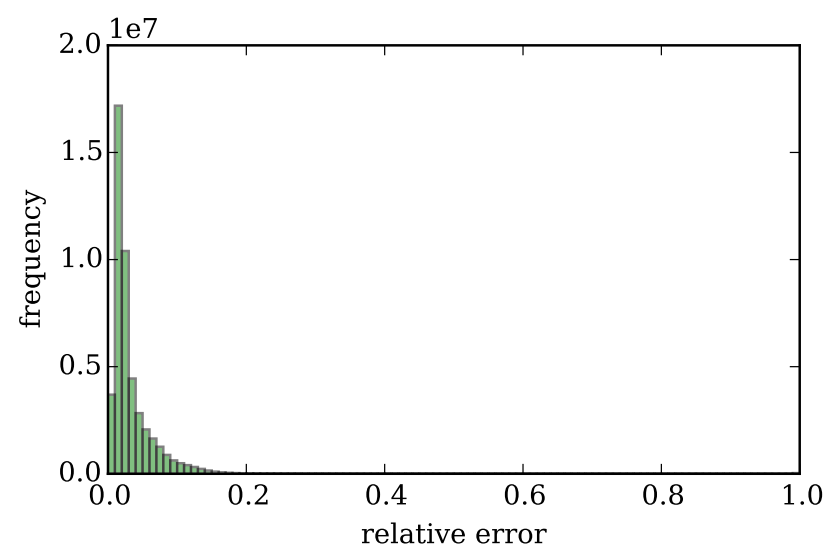

(a) Cartesian mesh

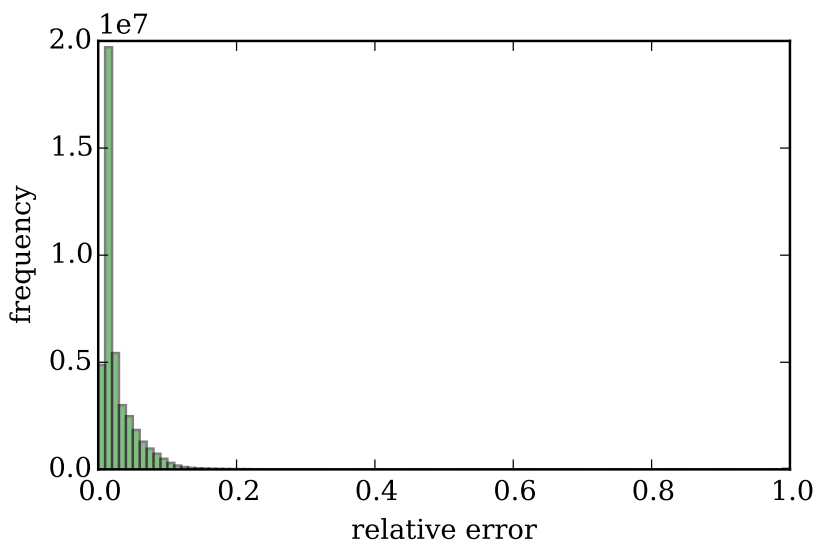

(b) Tetrahedral mesh

Figure 4: Distribution of relative errors for all position/energy phase space bins using Cartesian and tetrahedral mesh. Phase space bins with no scores are assigned a relative error of 1. Phase space bins for neutron energies greater than the maximum neutron source energy (therefore having no scores) are not included in these plots.

implemented in the PyNE variance_reduction module. CADIS requires an adjoint flux distribution using the detector of interest (in this case the dosimeter) as an adjoint source. This adjoint photon flux distribution was obtained by using the AutomateD VAriaNce reducTion Generator (ADVANTG) 27] to drive the Denovo 3D $\mathrm{S}_{N}$ code [28. Transport was carried out using a $\mathrm{P}_{3}$ Legendre 


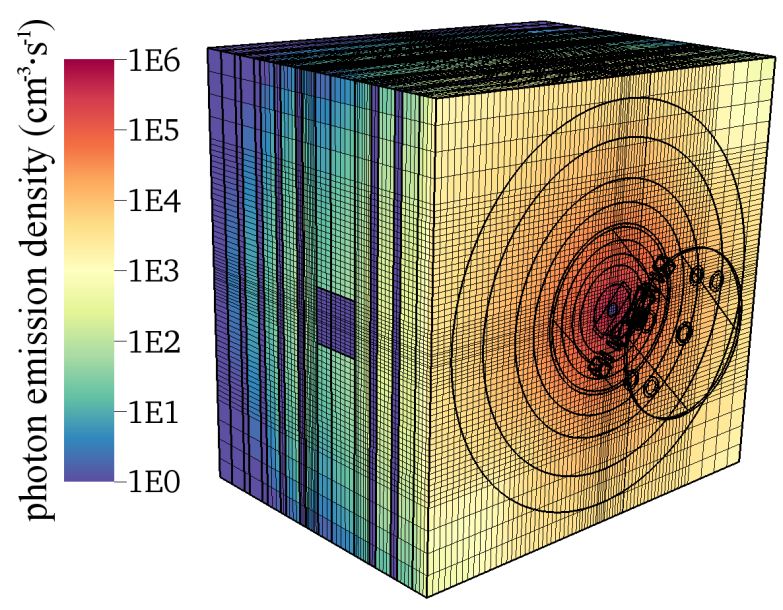

(a) Cartesian mesh

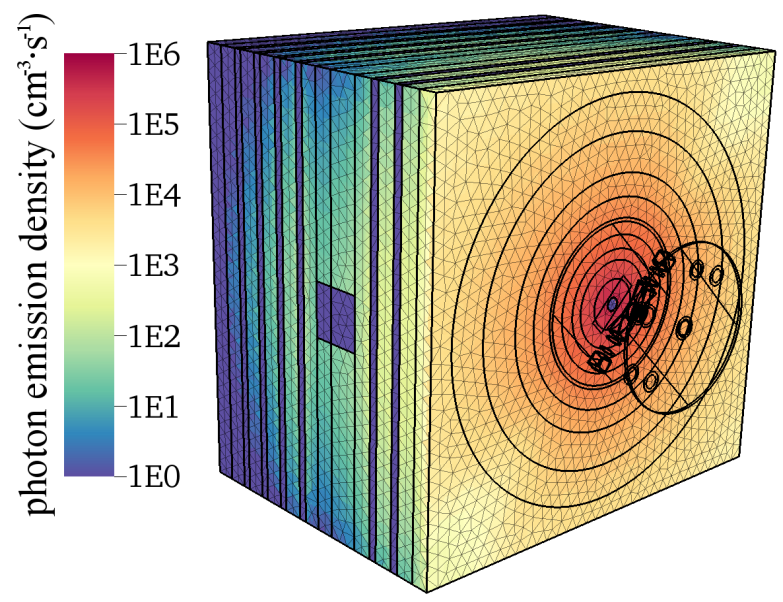

(b) Tetrahedral mesh

Figure 5: Total photon emission density distributions at the 1.22 hour decay time using Cartesian and tetrahedral mesh. Thick black lines represent geometry cells and thin black lines represent mesh volume elements.

order, and a quadruple range quadrature set of order 16. DPLUS 27] 23group photon data was used for this transport. Consequently, R2S photon source sampling with CADIS was done with the DPLUS group structure, which required a separate ALARA calculation with this photon binning. Unbiased photon transport was also done with this 23-group structure to corroborate the 
dose rate results obtained with CADIS.

Examples of unbiased and CADIS photon emission density distributions are show in Figure 6. This figure shows that although the true photon intensity is highest nearest to the neutron source, the CADIS method causes the source sampling to be biased such that simulated particles are more likely to be born around the central cavity (where the dosimeter is housed), as desired. The use of CADIS increased the MC Figure of Merit (FOM) relative to analog at all decay times, with modest speedups ranging from $2.1-6.6$. The FNG-ITER problem is not well-suited for showcasing the efficacy of photon variance reduction methods because the photon detector is in close proximity to the most activated regions. More significant performance enhancements enabled by the CADIS method are demonstrated in Section 4

For all photon transport simulations, $2 \cdot 10^{8}$ particles were simulated and ANSI/ANS-6.1.1-1977 [29] flux-to-dose-rate conversion factors were used to obtain final dose rate results. This choice of flux-to-dose-rate conversion factors facilitates a more direct comparison to previously published results from MCR2S [3] and MCFISP [16] which also used these factors. For all photon transport cases, final dose rate results had photon transport relative errors less than $0.7 \%$ as reported by DAG-MCNP5. Figure 7 shows the ratio of computational (C) to experimental (E) results obtained using PyNE R2S with Cartesian and tetrahedral mesh, and biased and unbiased photon source sampling. Results from several other R2S/D1S implementations are also included. Some systematic discrepancy between computational and experimental results is expected due to the use of spatial and energy discretization, the approximate irradiation scenario used, and error in the nuclear transport and activation data. Each of the results from other implementations shows discrepancies of similar magnitude at some decay times, and understanding these discrepancies is an ongoing effort throughout the community. The spread in computational results from PyNE R2S, MCR2S, and MCFISP observed at all decay times is likely due to different geometry discretizations and different degrees of convergence in transport simulations. Discrepancies between these results and results from R2Smesh are 
additionally accounted for by the use of ICRP-74 flux-to-dose-rate conversion factors [30] for R2Smesh photon transport [31].

PyNE R2S achieved satisfactory agreement with experimental results. Using the 24-group photon group structure, the maximum discrepancy with experimental results was $18.6 \%$ with Cartesian mesh and $20.4 \%$ with tetrahedral mesh. Tetrahedral results are systematically higher than Cartesian results due to the shape of the mesh volume elements in the region surrounding the central cavity: tetrahedral elements have center of masses closer to the dosimeter, so photons emitted from these mesh volume elements see less attenuation on average, resulting in slightly higher dose rates.

Using the 23-group DPLUS photon group structure, statistical agreement between the unbiased and CADIS results was achieved, thereby validating the biased sampling mode. These results also conform closely to the PyNE R2S 24-group Cartesian results (within 1.6\%) which suggests that the difference in group structure has a minimal affect in calculated dose rates in this case.

\section{CADIS Performance Demonstration}

The ability to perform mesh-based source biasing allows for the application of the CADIS method to R2S photon transport for target-based problems and FWCADIS for global variance reduction problems. In this section, the performance of the CADIS method is demonstrated with a series of target-based problems, each of which are more challenging than the standard FNG-ITER benchmark problem. Using the FNG photon geometry, four additional detector locations were chosen, represented as green circles in Figure 8 (a-d). These photon detector locations make the problem more challenging because they are much further from the neutron source. Each detector is an MCNP5 F5 point detector tally, modified with ANSI/ANS-6.1.1-1977 flux-to-dose-rate conversion factors.

Using the same procedure described in Section 3, CADIS weight windows and biased sources were calculated for the 19.8 day decay time for each of the four detectors. The ratios of biased to unbiased source densities for photon 
group $10(0.7-1.0 \mathrm{MeV})$ are shown in Figure 8 An example of the biased and unbiased source density distribution for point 2 is shown in Figure 9. In all cases it is apparent that the CADIS method successfully increases the probability of particle birth in the region near the detector and decreases the birth probability in regions far from the detector which are unlikely to produce scoring particles.

Photon transport was then done in analog and with the CADIS weight windows and source biasing parameters for each of the four detector locations. With CADIS, $2 \cdot 10^{8}$ particles were simulated for each detector location. For the ana$\log$ simulations, $2 \cdot 10^{8}$ particles were simulated for point 1 and $8 \cdot 10^{10}$ particles were simulated for points $2-4$. This was done in order to improve statistics for points $2-4$, which constitute significantly more difficult transport problems. For each of the transport problems, the MC FOM was recorded, as reported by DAG-MCNP5. The FOM is given by

$$
\mathrm{FOM}=\frac{1}{R^{2} T},
$$

where $R$ is the relative uncertainty of a tallied result and $T$ is the computer processor time. The increase in efficiency gained by using CADIS weight windows and source biasing is quantified by the speedup as given by:

$$
\text { speedup }=\frac{\text { FOM }_{\mathrm{CADIS}}}{\mathrm{FOM}_{\text {analog }}} .
$$

Dose rate results with and without CADIS and the calculated speedups are shown in Table 1. These results show that at all four detector locations statistically equivalent results were obtained with the two methods and that the CADIS method provided a significant speedup: as high as $8.5 \cdot 10^{5}$. Speedups of this magnitude will greatly reduce the computational resources necessary to obtain dose rate results for production-level problems.

\section{Conclusion}

PyNE R2S is a novel Cartesian- and tetrahedral-mesh-based R2S workflow that operates directly on CAD geometry and supports photon transport with 


\begin{tabular}{|c|r|r|r|}
\hline point & CADIS result $(\mu \mathrm{Sv} / \mathrm{h})$ & analog result $(\mu \mathrm{Sv} / \mathrm{h})$ & speedup \\
\hline 1 & $3.317 \pm 0.002 \cdot 10^{-2}$ & $3.31 \pm 0.01 \cdot 10^{-2}$ & $2.6 \cdot 10^{1}$ \\
\hline 2 & $6.42 \pm 0.01 \cdot 10^{-5}$ & $6.4 \pm 0.4 \cdot 10^{-5}$ & $5.6 \cdot 10^{5}$ \\
\hline 3 & $4.858 \pm 0.006 \cdot 10^{-3}$ & $4.92 \pm 0.05 \cdot 10^{-3}$ & $2.1 \cdot 10^{4}$ \\
\hline 4 & $2.603 \pm 0.005 \cdot 10^{-5}$ & $2.5 \pm 0.2 \cdot 10^{-5}$ & $8.5 \cdot 10^{5}$ \\
\hline
\end{tabular}

Table 1: Dose rate results at the 19.8 day decay time using analog and CADIS photon transport and the resulting speedups for the four points indicated in Figure 8 Reported uncertainties are the statistical uncertainties from MC photon transport.

advanced variance reduction. These new capabilities will reduce both the human and computational resources necessary to estimate the SDR for FES. PyNE R2S has been validated with the FNG-ITER shutdown dose rate benchmark problem using Cartesian and tetrahedral meshes with unbiased and CADIS photon sampling. Satisfactory agreement with experimental results was achieved, with all PyNE R2S results within 20.4\% of the experimental values. Speedups as high as $8.5 \cdot 10^{5}$ relative to analog transport were achieved using the CADIS method with problems using the FNG geometry. Future work will involve developing advanced variance reduction techniques for R2S neutron transport to further reduce the computational resources necessary for SDR analysis. Work is also underway to determine how uncertainty propagates through R2S calculations in order to quantify the uncertainty in photon dose rate results introduced from uncertainties in radiation transport results, nuclear data, and the mesh resolution. Understanding this uncertainty will be instrumental in reconciling discrepancies between experimental and computational results.

\section{Acknowledgments}

This work was funded in part by project DE-FG02-99ER54513 from the U.S. DOE Office of Fusion Energy Sciences and by the U.S. Nuclear Regulatory Commission Fellowship program. This research was performed using the compute resources and assistance of the UW-Madison Center For High Throughput 
Computing (CHTC) in the Department of Computer Sciences. The CHTC is supported by UW-Madison and the Wisconsin Alumni Research Foundation, and is an active member of the Open Science Grid, which is supported by the

350 National Science Foundation and the U.S. Department of Energy's Office of Science. 


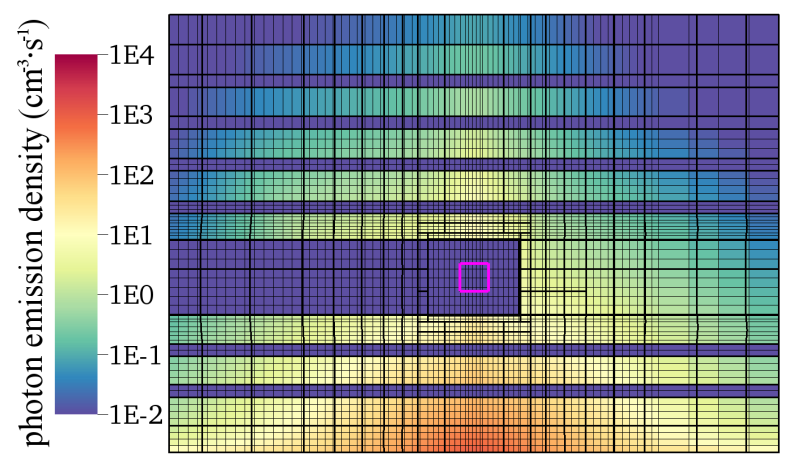

(a) Photon emission density, unbiased.

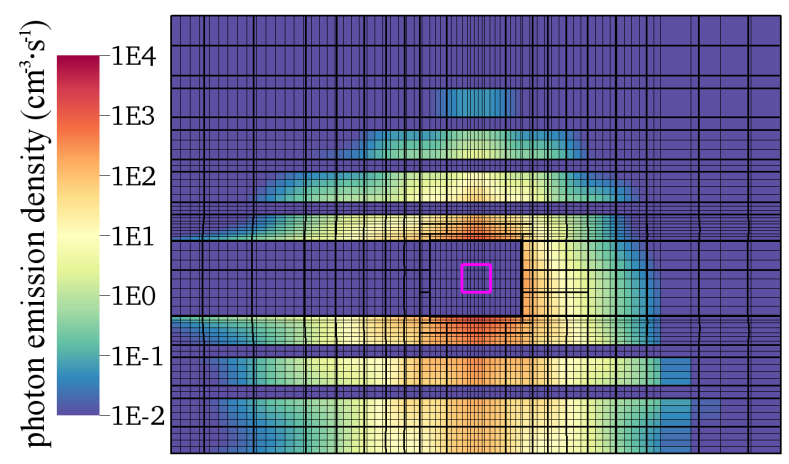

(b) Photon emission density, CADIS.

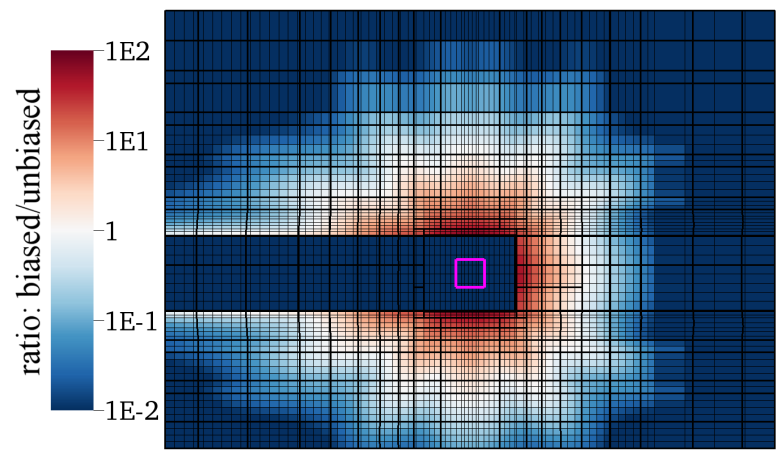

(c) Ratio of biased to unbiased photon emission densities.

Figure 6: Unbiased and biased photon emission density distributions and the ratio of the two for photon group $10(0.7-1.0 \mathrm{MeV})$ at the 19.8 day decay time. Plots show an $x-y$ slice of the FNG photon geometry at $z=-2.705 \mathrm{~cm}$ (through the center of the dosimeter, as seen in Figure 2(b)). Thick black lines represent geometry cells and thin black lines represent mesh volume elements. The pink square represents the outline of the dosimeter. 


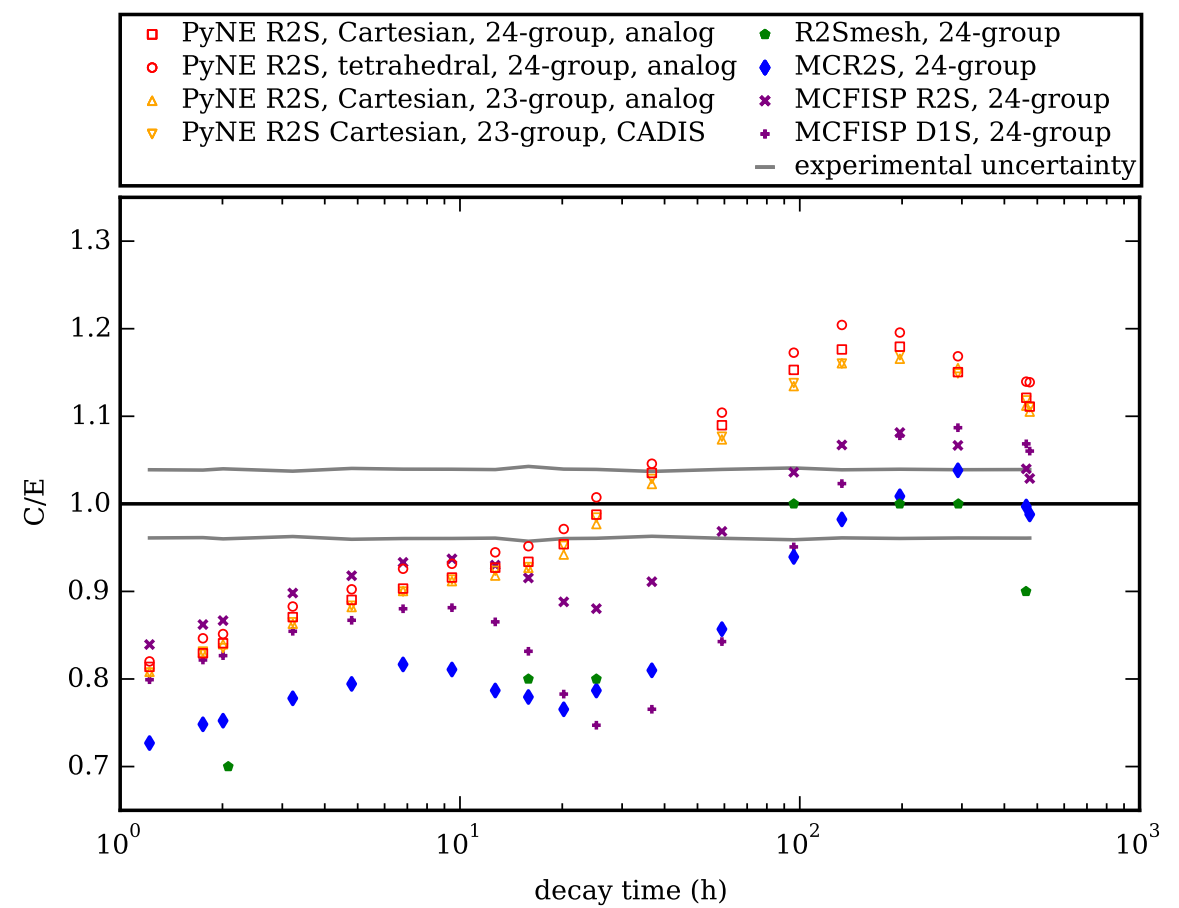

Figure 7: Ratio of calculated dose rates (C) to experimental dose rates (E), for the PyNE R2S workflow, MCR2S (results estimated from published plot) 3], R2Smesh 31, and MCFISP [16]. For the PyNE R2S results the relative errors in photon dose rates were all less than $0.7 \%$ as reported by DAG-MCNP5. 


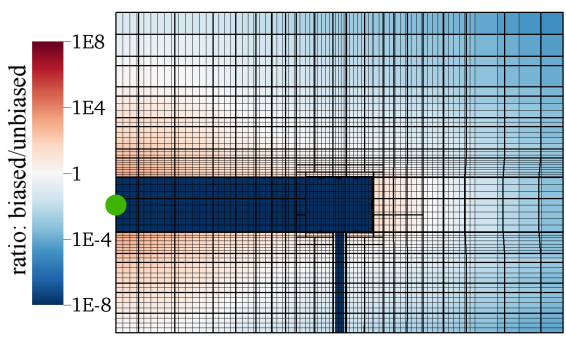

(a) Point 1

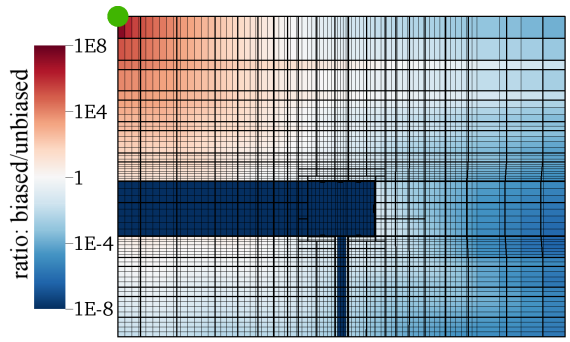

(b) Point 2

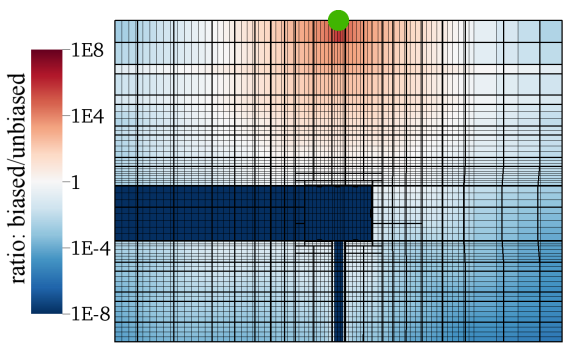

(c) Point 3

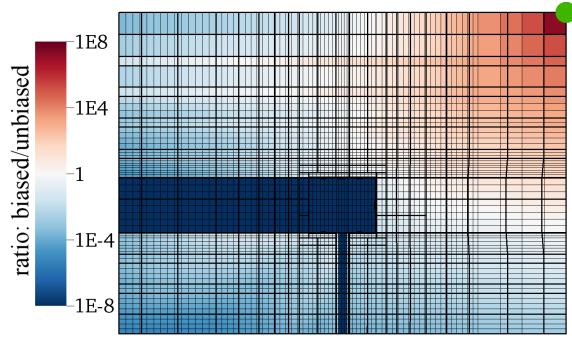

(d) Point 4

Figure 8: Ratio of biased to unbiased source densities for photon group $10(0.7-1.0 \mathrm{MeV})$ at the 19.8 day decay time for detectors located in four locations. Plots show an $x-y$ slice at $z=0 \mathrm{~cm}$. Detectors, represented as green circles, are MCNP5 F5 point detector tallies modified with ANSI/ANS-6.1.1-1977 flux-to-dose-rate conversion factors. 


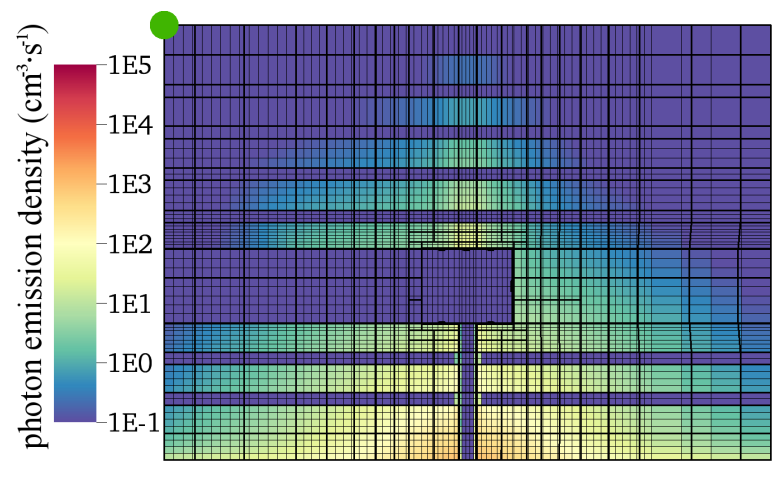

(a) Photon emission density, unbiased.

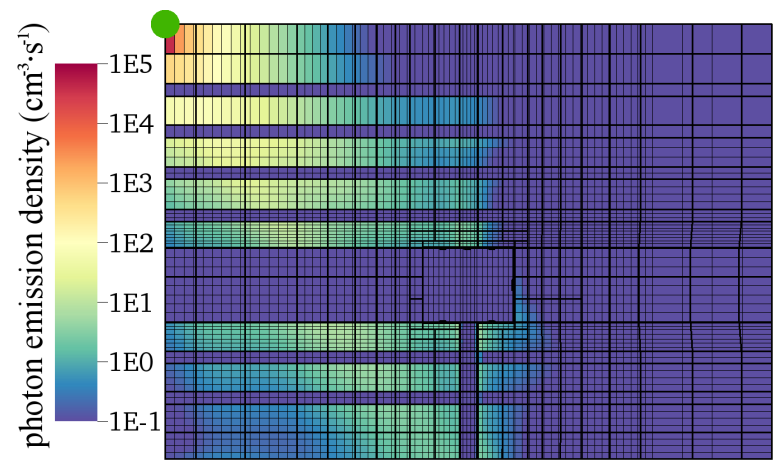

(b) Photon emission density, CADIS.

Figure 9: Biased and unbiased source densities for photon group $10(0.7-1.0 \mathrm{MeV})$ at the 19.8 day decay time for detector located at point 2. This plot shows an $x-y$ slice at $z=0 \mathrm{~cm}$. The detector, represented as a green circle, is an MCNP5 F5 point detector tally modified with ANSI/ANS-6.1.1-1977 flux-to-dose-rate conversion factors. 


\section{References}

[1] ITER Organization, ITER - the way to new energy, 2015. URL: http: //iter.org.

355 [2] Y. Chen, U. Fischer, Rigorous MCNP based shutdown dose rate calculations: Computational scheme, verification calculations and application to ITER, Fusion Engineering and Design 63-64 (2002) 107-114.

[3] A. Davis, R. Pampin, Benchmarking the MCR2S system for high-resolution activation dose analysis in ITER, Fusion Engineering and Design 85 (2010) $87-92$.

[4] T. J. Tautges, P. P. H. Wilson, J. Kraftcheck, B. F. Smith, D. L. Henderson, Acceleration techniques for direct use of CAD-Based geometries in Monte Carlo radiation transport, in: International Conference on Mathematics, Computational Methods \& Reactor Physics (M\&C 2009), American Nuclear Society, Saratoga Springs, NY, 2009.

[5] T. J. Tautges, R. Meyers, K. Merkley, C. Stimpson, C. Ernst, MOAB: A Mesh-Oriented Database, Technical Report, Sandia National Laboratories, 2004.

[6] M. E. Sawan et al., Application of CAD-neutronics coupling to geometrically complex fusion systems, in: Proc. 23rd Symp. Fusion Engineering (SOFE), San Diego, California, 2009.

[7] P. Sauvan, J. Catalán, F. Ogando, R. Juárez, J. Sanz, Development of the R2SUNED code system for shutdown dose rate calculations, IEEE Transactions on Nuclear Science 63 (2016) 375-384.

[8] D. Pelowitz, MCNP6 User's Manual Version 1.0, Technical Report LACFP-13-00634 Rev 0, Los Alamos National Laboratory, 2013.

[9] T. Eade, D. Stonell, A. Turner, \{MCR2S $\}$ unstructured mesh capabilities for use in shutdown dose rate analysis, Fusion Engineering and Design (2015). 
[10] G. D. Sjaardema, T. J. Tautges, T. J. Wilson, S. J. Owen, T. D. Blacker, W. J. Bohnhoff, T. L. Edwards, J. R. Hipp, R. R. Lober, S. A. Mitchell, S. N. Laboratories, CUBIT mesh generation environment Volume 1: Users manual, Sandia National Laboratories, 1994.

[11] C. A. D'Angelo, P. P. Wilson, A. Davis, Comparison between unstructured mesh capabilities of DAGMCNP and MCNP6, Transactions of the American Nuclear Society 109 (2013) 717-719.

[12] A. Haghighat, J. C. Wagner, Monte Carlo variance reduction with deterministic importance functions, Progress in Nuclear Energy 42 (2003) $25-53$.

[13] J. C. Wagner, D. E. Peplow, S. W. Mosher, FW-CADIS method for global and regional variance reduction of Monte Carlo radiation transport calculations, Nuclear Science and Engineering 176 (2014) 37-57.

[14] A. M. Ibrahim, S. W. Mosher, T. M. Evans, D. E. Peplow, M. E. Sawan, P. P. H. Wilson, J. C. Wagner, ITER neutronics modeling using hybrid Monte Carlo/ $\mathrm{S}_{N}$ and CAD-based Monte Carlo methods, Nuclear Technology 175 (2011) 251-258.

[15] P. P. H. Wilson, H. Tsige-Tamirat, H. Y. Khater, D. L. Henderson, Validation of the ALARA activation code, Fusion Technology 34 (1998) 784-788.

[16] P. Batistoni, M. Angelone, L. Petrizzi, M. Pillon, H. Freiesleben, D. Richter, K. Seidel, S. Unholzer, Y. Chen, U. Fischer, Experimental Validation of Shut-Down Dose Rates, Final Report ITER TASK T-426, 2001.

[17] E. Biondo, A. Davis, A. Scopatz, P. P. H. Wilson, Rigorous two-step activation for fusion systems with PyNE, in: Proc. of the 18th Topical Meeting of the Radiation Protection \& Shielding Division of ANS, Knoxville, TN, 
[18] D. Moule, P. Wilson, Mesh generation methods for deterministic radiation transport codes, in: Annual Meeting, American Nuclear Society, Hollywood, FL, United States, 2011.

[19] J. C. Smith, S. H. Jacobson, An analysis of the alias method for discrete random-variate generation, INFORMS Journal on Computing 17 (2005) $321-327$.

[20] C. Rocchini, P. Cignoni, Generating random points in a tetrahedron, Journal of Graphics Tools 5 (2001) 200-202.

[21] I. Kodeli, E. Sartori, B. Kirk, SINBAD shielding benchmark experiments status and planned activities, in: ANS 14th Biennial Topical Meeting of the Radiation Shielding Protection and Shielding Division, 2006.

[22] UW Madison Computational Nuclear Engineering Research Group, MCNP2CAD, https://github.com/svalinn/mcnp2cad, 2015.

[23] M. Chadwick, P. Obložinský, M. Herman, N. Greene, R. McKnight, D. Smith, et. al., ENDF/B-VII.0: Next generation evaluated nuclear data library for nuclear science and technology, Nuclear Data Sheets 107 (2006) $2931-3060$.

[24] A. Davis, Comparison of global variance reduction techniques for Monte Carlo radiation transport simulations of ITER, Fusion Engineering and Design 86 (2011) 2698-2700.

[25] J. Kopecky, VALIDATION OF FENDL-3/A LIBRARY USING INTEGRAL MEASUREMENTS, Technical Report INDC(NED)-011, IAEA INDC International Nuclear Data Committee, Vienna, Austria, 2012.

[26] R. A. Forrest, FISPACT-2007: User Manual, Technical Report UKAEA FUS 534, Culham Science Centre, Oxfordshire, OX14 3DB, 2007.

[27] S. W. Mosher, A. M. Bevill, S. R. Johnson, A. M. Ibrahim, C. R. Daily, T. M. Evans, J. C. Wagner, J. O. Johnson, R. E. Grove, ADVANTG-An 
Automated Variance Reduction Parameter Generator, Technical Report ORNL/TM-2013/416, Oak Ridge National Laboratory, 2013.

[28] T. M. Evans, A. S. Stafford, R. N. Slaybaugh, K. T. Clarno, Denovo: A new three-dimensional parallel discrete ordinates code in SCALE, Nuclear Technology 171 (2010) 171-200.

[29] Neutron and gamma-ray flux-to-dose-rate factors, American Nuclear Society, ANSI Standard ANSI/ANS-6.1.1-1977, 1977.

[30] Conversion coefficients for use in radiological protection against external radiation, ICRP Publication 7426 (1996).

[31] M. Majerle, D. Leichtle, U. Fischer, A. Serikov, Verification and validation of the R2Smesh approach for the calculation of high resolution shutdown dose rate distributions, Fusion Engineering and Design 87 (2012) 443-447. 


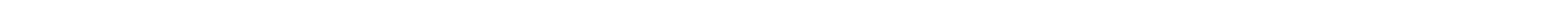

